\title{
Discrepancies between Conventional Multiscale Entropy and Modified Short-Time Multiscale Entropy of Photoplethysmographic Pulse Signals in Middle- and Old- Aged Individuals with or without Diabetes
}

\author{
Gen-Min Lin ${ }^{1,2,3, *}$, Bagus Haryadi ${ }^{1,4}$, Chieh-Ming Yang ${ }^{1}$, Shiao-Chiang Chu ${ }^{1}$, \\ Cheng-Chan Yang ${ }^{1,5}$ and Hsien-Tsai $\mathrm{Wu}^{1, *}$ \\ 1 Department of Electrical Engineering, National Dong-Hwa University, Hualien 97401, Taiwan; \\ 810523004@gms.ndhu.edu.tw (B.H.); j82887@gmail.com (C.-M.Y.); 610523004@gms.ndhu.edu.tw (S.-C.C.); \\ joseph9204@yahoo.com.tw (C.-C.Y.) \\ 2 Department of Medicine, Hualien Armed Forces General Hospital, Hualien 970, Taiwan \\ 3 Departments of Medicine, Tri-Service General Hospital, National Defense Medical Center, Taipei 114, Taiwan \\ 4 Department of Physics, Universitas Ahmad Dahlan, Yogyakarta 55164, Indonesia \\ 5 Department of Traditional Chinese Medicine, Buddhist Tzu-Chi General Hospital, Hualien 970, Taiwan \\ * Correspondence: farmer507@yahoo.com.tw (G.-M.L.); hsientsaiwu@gmail.com (H.-T.W.); \\ Tel.: +886-862-0601 (G.-M.L.); +886-3-863-4099 (H.-T.W.); \\ Fax: +886-3-826-1370 (G.-M.L.); +886-3-863-4060 (H.-T.W.)
}

Academic Editor: Herbert Jelinek

Received: 2 February 2017; Accepted: 16 March 2017; Published: 18 March 2017

\begin{abstract}
Multiscale entropy (MSE) of physiological signals may reflect cardiovascular health in diabetes. The classic MSE (cMSE) algorithm requires more than 750 signals for the calculations. The modified short-time MSE (sMSE) may have inconsistent outcomes compared with the cMSE at large time scales and in a disease status. Therefore, we compared the cMSE of 1500 ( $\mathrm{CMSE}_{1500}$ ) consecutive and 1000 photoplethysmographic (PPG) pulse amplitudes with the sMSE of 500 PPG $\left(\mathrm{sMSE}_{500}\right)$ pulse amplitudes of bilateral fingertips among middle- to old-aged individuals with or without type 2 diabetes. We discovered that $\mathrm{cMSE}_{1500}$ had the smallest value across scale factors 1-10, followed by $\mathrm{cMSE}_{1000}$, and then sMSE $\mathrm{S}_{500}$ in both hands. The $\mathrm{cMSE}_{1500}, \mathrm{CMSE}_{1000}$ and sMSE 500 did not differ at each scale factor in both hands of persons without diabetes and in the dominant hand of those with diabetes. In contrast, the sMSE $_{500}$ differed at all scales 1-10 in the non-dominant hand with diabetes. In conclusion, autonomic dysfunction, prevalent in the non-dominant hand which had a low local physical activity in the person with diabetes, might be imprecisely evaluated by the SMSE; therefore, using more PPG signal numbers for the cMSE is preferred in such a situation.
\end{abstract}

Keywords: multiscale entropy; photoplethysmographic wave amplitudes; diabetes

\section{Introduction}

Costa et al. proposed conventional multiscale entropy (cMSE) for evaluations of the complexity of physiological signals [1]. In the cMSE algorithm, the original time series is coarse-grained and the sample entropy $\left(S_{E}\right)$ for each coarse-grained time series is calculated to quantify regularity at different time scales. The decimation procedure shortens the coarse-grained time series length by a factor of $\tau$. Therefore, the coarse-grained time series at large scales may lead to an imprecise estimation of $S_{E}$ [2]. In addition, the $S_{E}$ may be invalid as long as no template vectors are matched to one another. Inaccurate or invalid $S_{E}$ can reduce the reliability in distinguishing time series generated by different systems. 
These problems usually bring challenges of the cMSE algorithm at large-scale factors. Costa et al. suggested that to avoid an error at large-scale factors, the measured signal numbers had to be greater than 750 [3].

For a physiological signal such as pulse rate, which has an average of 60-100 beats per minute at rest, the signals usually require at least $20 \mathrm{~min}$ recording to obtain sufficient data for the CMSE analysis. Accordingly, a few modified MSE algorithms were developed for clinical applications with short-term data [2,4-6]. Wu et al. proposed the composite MSE and the refined one using a short-term signal series to compare the accuracy and validity of their modified MSE algorithms with the CMSE, whereas there were no data for the clinical application to the normal subjects and the diseased patients $[2,4]$. In contrast, Choi developed another modified MSE algorithm using 1000 electroencephalographic wave signals to compare the accuracy with the cMSE in normal, interictal, and ictal conditions [5]. Although the results of the modified MSE were optimal to differentiate the three conditions, the signal points were greater than 750 as suggested and might not be fit for the so-called "short-time" MSE. With regard to the modified short-duration MSE (sMSE) proposed by Chang et al., the sMSE of 600 peripheral arterial pulse wave velocity (PWV) signals was in line with the cMSE of 1000 PWV signals in healthy individuals and in those with diabetes [6]. Furthermore, Yang et al. used the sMSE of 350-400 electrocardiographic R-R intervals to evaluate the change of peripheral blood flow before and after acupuncture in healthy subjects [7].

In a disease status, such as heart failure, the value of the cMSE is theoretically reduced at large scales and there may be a great concern for using sMSE in such a situation. Wu et al. also found that the cMSE of fingertip photoplethysmographic (PPG) pulse amplitudes in those with diabetes may be lower than that of the unaffected person [8]. Therefore, the aim of our study was to compare the sMSE of PPG pulse amplitudes of bilateral fingertips with the cMSE in healthy subjects and in those with type 2 diabetes.

\section{Methods}

\subsection{Study Population}

Between July 2009 and October 2010, 113 right-hand dominant, middle- to old-aged men and women were prospectively enrolled for PPG examinations of their bilateral index fingertips in the Ministry of Health and Welfare Hualien Hospital. Of this population, 11 participants were excluded for a history of cardiovascular disease including coronary heart disease, ischemic stroke, peripheral vascular disease, heart failure, atrial fibrillation, and permanent pacemaker implantation, leaving a sample of 102 individuals for the MSE analysis. Diabetes mellitus was defined as fasting glucose $\geq 126 \mathrm{mg} / \mathrm{dL}$, or glycated hemoglobin $(\mathrm{HbA} 1 \mathrm{c})>6.5 \%$, or the use of hypoglycemic medications. Of these, 49 participants did not have diabetes mellitus (the unaffected group), and 53 had type 2 diabetes. Since age, sex, and body weight might affect the MSE index, we matched these variables at baseline and selected 70 individuals $(68.6 \%)$ from the initial study sample (35 from the unaffected and 35 from the diabetes groups, respectively) to perform a case-control comparison. This study was reviewed and approved by the Institutional Review Board of Hualien Hospital and National Dong-Hwa University. Each patient signed an informed consent.

\subsection{Study Protocol}

All measurements and procedures were performed in the morning (i.e., 8:30-10:30 a.m.). Demographic, anthropometric, and laboratory data for the analyses were obtained at the visit. Body mass index was defined as weight divided by height squared $\left(\mathrm{kg} / \mathrm{m}^{2}\right)$. Resting blood pressure was measured once over left arm of the supine participants by an automated oscillometric device (Microlife BP3AG1, Taipei, Taiwan) with an appropriate cuff size. Cholesterol and triglycerides concentrations were measured from blood samples obtained after a $12 \mathrm{~h}$ fast. 
The participants were asked to refrain from caffeine-containing beverages and theophyllinecontaining medications for $12 \mathrm{~h}$ before each hospital visit. In addition, to minimize potential erroneous readings from the infrared sensors arising from involuntary vibrations of the examinees and a decreased environmental temperature that tends to cause constriction of the peripheral vessels, all participants underwent blood sampling before data acquisition and were allowed to relax in a supine position for $10 \mathrm{~min}$ in a quiet room with temperature control at $26 \pm 1{ }^{\circ} \mathrm{C}$. The PPG infrared sensors were simultaneously applied to bilateral index fingertips of each participant for the acquisition of data for $30 \min [8,9]$.

\subsection{Calculation of Bilateral Fingertips PPG Pulse Amplitude Series}

After being processed through an analog-to-digital converter (USB-6009 DAQ, National Instruments, Austin, TX, USA) at a sampling frequency of $500 \mathrm{~Hz}$, the digitized PPG signals were stored on a computer and analyzed by the Matlab 7.7 software (MathWorks, Natick, MA, USA) [8]. PPG pulse amplitude was defined as the systolic peak or the distance between peak and trough of a pulse waveform (Figure 1). We retrieved consecutive 1500 PPG pulse amplitudes of left hand shown as $\operatorname{PPGA}_{L}=\left\{\operatorname{PPGA}_{L}(1), \operatorname{PPGA}_{L}(2), \operatorname{PPGA}_{L}(3), \ldots, \operatorname{PPGA}_{L}(n)\right\}$ and those of right hand as $\operatorname{PPGA}_{R}=\left\{\operatorname{PPGA}_{R}(1), \operatorname{PPGA}_{R}(2), \operatorname{PPGA}_{R}(3), \ldots, \operatorname{PPGA}_{R}(n)\right\}$ from each participant simultaneously.
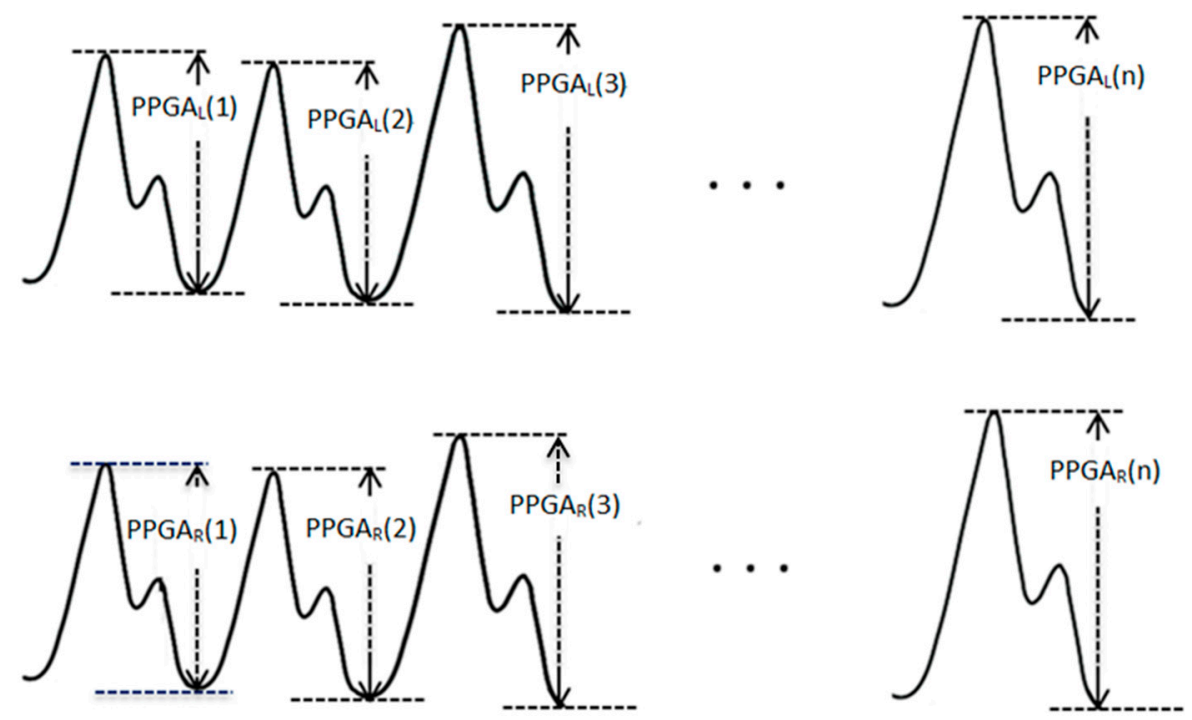

Figure 1. PPGA of left hand $\left(P P G A_{L}\right)$ and right hand $\left(P P G A_{R}\right)$ were simultaneously acquired from PPGA(1) to PPGA $(n=500,1000$, or 1500$)$.

\subsubsection{Detrending of PPGA Series Signal}

Empirical mode decomposition (EMD), proposed by Huang et al. [10,11], is a time space method to extract final signals regressed to the zero mean after the noises are removed from data generated in nonlinear and non-stationary processes. EMD was used to partition the $\left\{\operatorname{PPGA}_{\mathrm{L}}(i)\right\}$ and $\left\{\operatorname{PPGA}_{\mathrm{R}}(j)\right\}$ series, thereby eliminating the trend from the original series. Then the $\left\{\operatorname{PPGA}_{L}(i)\right\}$ and $\left\{\operatorname{PPGA}_{R}(j)\right\}$ series were normalized as shown in (1). In these equations, $\mathrm{SD}_{\mathrm{PPGA}_{L}}$ and $\mathrm{SD}_{\mathrm{PPGA}_{R}}$ represent the standard deviations of series $\left\{\operatorname{PPGA}_{L}(i)\right\}$ and $\left\{\operatorname{PPGA}_{R}(j)\right\}$, respectively. $\overline{P P G A}_{L}$ and $\overline{\text { PPGA }_{R}}$ represent the mean of series $\left\{\operatorname{PPGA}_{\mathrm{L}}(i)\right\}$ and $\left\{\operatorname{PPGA}_{\mathrm{R}}(j)\right\}$, respectively. Complexity analysis was performed on the normalized results, $n$ PPGA $_{\mathrm{L}}(i)$ and $n \mathrm{PPGA}_{\mathrm{R}}(j)$.

$$
\begin{aligned}
n \operatorname{PPGA}_{\mathrm{L}}(i) & =\frac{\operatorname{PPGA}_{\mathrm{L}}(i)-\overline{\mathrm{PPGA}_{\mathrm{L}}}}{\operatorname{SD}_{\mathrm{PPGA}}} \\
n \operatorname{PPGA}_{\mathrm{R}}(j) & =\frac{\operatorname{PPGA}_{\mathrm{R}}(j)-\overline{\operatorname{PPGA}_{R}}}{\mathrm{SD}_{\mathrm{PPGA}_{R}}}
\end{aligned}
$$




\subsubsection{Coarse-Grained Process and $S_{E}$}

Multiple coarse-grained time series are constructed by averaging the data points within non-overlapping windows of increasing length, $\tau$ (i.e., the scale factor), as follows:

$$
y_{j}^{(\tau)}=\frac{1}{\tau} \sum_{i=(j-1) \tau+1}^{j \tau} x_{i}, 1 \leq j \leq \frac{N}{\tau}
$$

Thus, the length of each coarse-grained time series is $N / \tau$. $S_{E}$ is then computed for each new coarse-grained time series $\left\{y_{j}^{(\tau)}\right\}$, and plotted as a function of the scale factor [12].

\subsubsection{The cMSE of Bilateral Fingertips PPG Series Signal}

cMSE consists of two main procedures, namely coarse-graining and calculation of $S_{E}$ for each coarse-grained time series. First, the computation of $S_{E}$ comprises:

(a) Define the data series $x(n)$ with length $N$ and the two parameters of $m$ and $r$ (where $m=$ Embedded dimension of the vector; $r=$ tolerance). $N=1000$ and 1500 for $n \operatorname{PPGA}_{L}(i)$ and $n \operatorname{PPGA}_{R}(j)$, respectively. In addition, $m=2$ and $r=0.15$ were set according to previous Costa et al. study recommendation [13].

(b) Define $N-m+1$ vectors, each of size $m$, composed as follows:

$$
u_{m}(i)=\left\{x_{i}, x_{i+1}, \ldots, x_{i+m-1}\right\}, 1 \leq i \leq N-m+1
$$

(c) Define $\mathrm{d}\left[u_{m}(i), u_{m}(j)\right]$ as the maximum value: $\mathrm{d}\left[u_{m}(i), u_{m}(j)\right]=\max \left\{\left|x_{i+k}-x_{j+k}\right|: 0 \leq k \leq m-1\right\}$ $(i \neq j)$. Calculate the number of $\mathrm{d}\left[u_{m}(i), u_{m}(j)\right]$ within distance $r$ and calculate the ratio of the number to the total $N-m$ for each value of $i \leq N-m+1$ and an average to all points is defined as:

$$
C_{m}(r)=\frac{1}{N-m+1} \sum_{i=1}^{N-m+1} \ln \frac{n_{i}{ }^{m}}{N-m+1}
$$

(d) Increase the embedded dimension to $m+1$, gives:

$$
C_{m+1}(r)=\frac{1}{N-m} \sum_{i=1}^{N-m} \ln \frac{n_{i}^{(m+1)}}{N-m}
$$

(e) Therefore, $S_{E}$ is defined as:

$$
S_{E}(m, r, N)=C_{m}(r)-C_{m+1}(r)
$$

\subsubsection{The sMSE of Bilateral Fingertips PPG Series Signal}

The cMSE method adopts the original time series for computation, whereas the sMSE method takes advantage of the time lags from a number of time series generated from the original one. Through altering the number of lag from 0 to $L$ (where $L=\tau-1, \tau=$ coarse-grained scale factor) on the native time series which we selected $N=500$ for bilateral fingers PPG pulse amplitudes (7), a new time series, $T^{(P)}$, can be obtained (8). Thus, the number of new time series generated is $L+1$ :

$$
\begin{gathered}
T_{N}=\left\{X_{1}, X_{2}, \ldots, X_{N-1}, X_{N}\right\} \\
T^{(P)}=\left\{X_{k}, X_{k+1}, X_{k+2}, \ldots, X_{N-1}, X_{N}\right\}, k=p+1, p=0,1,2, \ldots, L
\end{gathered}
$$

The $L+1$ time series acquired then undergo coarse-grained processing with a scale factor $\tau$ (9), giving the time series of $y^{(p)(\tau)}$. Hence: 


$$
y_{j}^{(p)(\tau)}=\frac{1}{\tau} \sum_{k=(j-1) \tau+1+p}^{j \tau+p} X_{k}, 1 \leq j \leq\left\lfloor\frac{N-P}{\tau}\right\rfloor, p=0,1,2, \ldots, L
$$

The $L+1 y^{(p)(\tau)}$ are then subjected to $S_{E}$ computation and averaged, giving sMSE of scale factor $\tau$ in (10):

$$
\operatorname{sMSE}_{\tau}=\frac{1}{L+1} \sum_{p=0}^{L} S_{E}\left(y^{(p)(\tau)}\right)
$$

\subsubsection{The PPGA-Based cMSE and sMSE Index}

On analyzing PPG signals by using both the cMSE and sMSE algorithms, Costa et al. reported the association of $S_{E}$ of small scales $(\tau=1-5)$ with autonomic nervous activity and respiratory modulation [13]. Accordingly, the present study analyzed PPG signals using an overall MSE index (the average of scales 1-10, MSE $1-10$ ) and by dividing the MSE into small scales (the average of scales 1-5, MSE $1-5$ ) and large scales (scales 6-10, $\mathrm{MSE}_{6-10}$ ) for inter-groups comparisons.

\subsection{Statistical Analysis}

The data are presented as mean \pm standard deviation (SD). The continuous variables between the groups were compared using two-tailed $t$ test and categorical variables between the groups were compared using chi-square test or Fisher's exact test. The values between $\mathrm{SMSE}_{500}$ and each cMSE of PPG pulse amplitudes were compared using one-sample $t$ test. A $p<0.05$ was considered statistically significant. The SPSS software (Version 14.0, SPSS Inc., Chicago, IL, USA) was adopted for all statistical analyses.

\section{Results}

The baseline characteristics of the age-, sex-, and weight-matched two groups are shown in Table 1. As compared with participants in the unaffected group, those in the diabetes group had a faster pulse rate and higher $\mathrm{HbA1c}$ levels.

Table 1. Baseline characteristics of the unaffected and diabetic participants.

\begin{tabular}{cccc}
\hline & Unaffected $(\boldsymbol{n}=\mathbf{3 5 )}$ & Diabetes $(\boldsymbol{n}=\mathbf{3 5 )}$ & $\boldsymbol{p}$-Value \\
\hline Male, $\%$ & $23(51.43)$ & $24(54.29)$ & 0.811 \\
Age, year & $59.74(8.19)$ & $60.2(6.58)$ & 0.798 \\
Height, $\mathrm{m}$ & $161.62(8.48)$ & $169.82(50.45)$ & 0.360 \\
Weight, kg & $67.82(10.22)$ & $70.61(14.73)$ & 0.372 \\
Waist circumference, cm & $88.47(7.98)$ & $90.94(12.39)$ & 0.336 \\
Pulse rate, beats/min & $73.42(8.68)$ & $81.34(10.93)$ & 0.002 \\
Systolic BP, mmHg & $127.94(18.74)$ & $123.91(16.13)$ & 0.349 \\
Diastolic BP, mmHg & $75.82(10.84)$ & $74.23(10.16)$ & 0.539 \\
HbA1c, \% & $6.00(0.39)$ & $8.74(1.74)$ & 0.000 \\
Total cholesterol, mg/dL & $188.15(35.48)$ & $182.06(37.28)$ & 0.506 \\
Triglyceride, mg/dL & $111.18(84.84)$ & $137.23(69.40)$ & 0.187 \\
HDL-C, mg/dL & $47.24(16.09)$ & $41.97(17.78)$ & 0.218 \\
\hline
\end{tabular}

Continuous variables are expressed as mean (SD) and categorical variables as number (percentage). Abbreviations: $\mathrm{BP}$, blood pressure; HbA1c, hemoglobin A1c; HDL-C, high-density lipoprotein cholesterol.

Table 2 shows the comparisons between the cMSE of 1500 PPG pulse amplitudes (cMSE 1500 ) and the sMSE of 500 PPG pulse amplitudes ( $\mathrm{sMSE}_{500}$ ) in the right dominant hand and left non-dominant hand of the individuals with or without diabetes.

The values of the $\mathrm{CMSE}_{1500}$ and those of the $\mathrm{SMSE}_{500}$ in both hands of the unaffected individuals did not differ at most scale factors 1-10, and neither did those of the right dominant hand (which means predominantly using one's right hand) of the individuals with diabetes. On the contrary, the values of 
$\mathrm{sMSE}_{500}$ were significantly greater than those of $\mathrm{cMSE}_{1500}$ in the non-dominant hand of the individuals with diabetes across all scale factors 1-10.

Table 2. A comparison between $\mathrm{CMSE}_{1500}$ and $\mathrm{SMSE}_{500}$ of right hand and left hand in the unaffected and diabetic participants at each scale factor. Data are presented as mean (standard error) of MSE. One-sample $t$-test was used to compare the values between $\mathrm{CMSE}_{1500}$ and $\mathrm{sMSE}_{500}$ in each group.

\begin{tabular}{|c|c|c|c|c|c|c|c|c|c|c|}
\hline & Scale 1 & Scale 2 & Scale 3 & Scale 4 & Scale 5 & Scale 6 & Scale 7 & Scale 8 & Scale 9 & Scale 10 \\
\hline \multicolumn{11}{|c|}{ Unaffected } \\
\hline \multicolumn{11}{|c|}{ right hand } \\
\hline $\mathrm{cMSE}_{1500}$ & $\begin{array}{c}1.28 \\
(0.07)\end{array}$ & $\begin{array}{c}1.24 \\
(0.05)\end{array}$ & $\begin{array}{c}1.23 \\
(0.05)\end{array}$ & $\begin{array}{c}1.29 \\
(0.05)\end{array}$ & $\begin{array}{c}1.41 \\
(0.05)\end{array}$ & $\begin{array}{c}1.50 \\
(0.06)\end{array}$ & $\begin{array}{c}1.57 \\
(0.06)\end{array}$ & $\begin{array}{c}1.61 \\
(0.06)\end{array}$ & $\begin{array}{c}1.62 \\
(0.06)\end{array}$ & $\begin{array}{c}1.69 \\
(0.06)\end{array}$ \\
\hline sMSE $_{500}$ & $\begin{array}{c}1.46 \\
(0.07)\end{array}$ & $\begin{array}{c}1.36 \\
(0.05)\end{array}$ & $\begin{array}{c}1.35 \\
(0.06)\end{array}$ & $\begin{array}{c}1.39 \\
(0.05)\end{array}$ & $\begin{array}{c}1.48 \\
(0.05)\end{array}$ & $\begin{array}{c}1.57 \\
(0.06)\end{array}$ & $\begin{array}{c}1.66 \\
(0.06)\end{array}$ & $\begin{array}{c}1.74 \\
(0.07)\end{array}$ & $\begin{array}{c}1.71 \\
(0.07)\end{array}$ & $\begin{array}{c}1.78 \\
(0.07)\end{array}$ \\
\hline$p$-value & 0.082 & 0.10 & 0.12 & 0.16 & 0.30 & 0.40 & 0.26 & 0.19 & 0.32 & 0.34 \\
\hline \multicolumn{11}{|c|}{ left hand } \\
\hline $\mathrm{cMSE}_{1500}$ & $\begin{array}{c}1.29 \\
(0.08)\end{array}$ & $\begin{array}{c}1.27 \\
(0.06)\end{array}$ & $\begin{array}{c}1.24 \\
(0.04)\end{array}$ & $\begin{array}{c}1.31 \\
(0.04)\end{array}$ & $\begin{array}{c}1.43 \\
(0.05)\end{array}$ & $\begin{array}{c}1.50 \\
(0.04)\end{array}$ & $\begin{array}{c}1.57 \\
(0.04)\end{array}$ & $\begin{array}{c}1.61 \\
(0.05)\end{array}$ & $\begin{array}{c}1.65 \\
(0.05)\end{array}$ & $\begin{array}{c}1.68 \\
(0.05)\end{array}$ \\
\hline sMSE $_{500}$ & $\begin{array}{c}1.39 \\
(0.06)\end{array}$ & $\begin{array}{c}1.39 \\
(0.06)\end{array}$ & $\begin{array}{c}1.40 \\
(0.06)\end{array}$ & $\begin{array}{c}1.41 \\
(0.07)\end{array}$ & $\begin{array}{c}1.52 \\
(0.06)\end{array}$ & $\begin{array}{c}1.60 \\
(0.06)\end{array}$ & $\begin{array}{c}1.70 \\
(0.07)\end{array}$ & $\begin{array}{c}1.73 \\
(0.07)\end{array}$ & $\begin{array}{c}1.78 \\
(0.08)\end{array}$ & $\begin{array}{c}1.79 \\
(0.07)\end{array}$ \\
\hline$p$-value & 0.30 & 0.14 & 0.044 & 0.22 & 0.25 & 0.21 & 0.11 & 0.17 & 0.17 & 0.18 \\
\hline \multicolumn{11}{|c|}{ Diabetes } \\
\hline \multicolumn{11}{|c|}{ right hand } \\
\hline $\mathrm{cMSE}_{1500}$ & $\begin{array}{c}1.24 \\
(0.07)\end{array}$ & $\begin{array}{c}1.13 \\
(0.06)\end{array}$ & $\begin{array}{c}1.11 \\
(0.06)\end{array}$ & $\begin{array}{c}1.13 \\
(0.06)\end{array}$ & $\begin{array}{c}1.18 \\
(0.05)\end{array}$ & $\begin{array}{c}1.25 \\
(0.05)\end{array}$ & $\begin{array}{c}1.30 \\
(0.06)\end{array}$ & $\begin{array}{c}1.34 \\
(0.05)\end{array}$ & $\begin{array}{c}1.38 \\
(0.06)\end{array}$ & $\begin{array}{c}1.39 \\
(0.06)\end{array}$ \\
\hline $\mathrm{sMSE}_{500}$ & $\begin{array}{c}1.45 \\
(0.09)\end{array}$ & $\begin{array}{c}1.30 \\
(0.07)\end{array}$ & $\begin{array}{c}1.26 \\
(0.07)\end{array}$ & $\begin{array}{c}1.23 \\
(0.07)\end{array}$ & $\begin{array}{c}1.33 \\
(0.07)\end{array}$ & $\begin{array}{c}1.38 \\
(0.07)\end{array}$ & $\begin{array}{c}1.44 \\
(0.08)\end{array}$ & $\begin{array}{c}1.53 \\
(0.09)\end{array}$ & $\begin{array}{c}1.52 \\
(0.08)\end{array}$ & $\begin{array}{c}1.53 \\
(0.08)\end{array}$ \\
\hline$p$-value & 0.080 & 0.078 & 0.16 & 0.33 & 0.14 & 0.21 & 0.20 & 0.086 & 0.15 & 0.21 \\
\hline \multicolumn{11}{|c|}{ left hand } \\
\hline $\mathrm{cMSE}_{1500}$ & $\begin{array}{c}1.12 \\
(0.08)\end{array}$ & $\begin{array}{c}1.07 \\
(0.07)\end{array}$ & $\begin{array}{c}1.05 \\
(0.06)\end{array}$ & $\begin{array}{c}1.08 \\
(0.06)\end{array}$ & $\begin{array}{c}1.16 \\
(0.06)\end{array}$ & $\begin{array}{c}1.25 \\
(0.06)\end{array}$ & $\begin{array}{c}1.31 \\
(0.07)\end{array}$ & $\begin{array}{c}1.32 \\
(0.07)\end{array}$ & $\begin{array}{c}1.37 \\
(0.07)\end{array}$ & $\begin{array}{c}1.41 \\
(0.08)\end{array}$ \\
\hline sMSE $_{500}$ & $\begin{array}{c}1.52 \\
(0.08)\end{array}$ & $\begin{array}{c}1.41 \\
(0.07)\end{array}$ & $\begin{array}{c}1.36 \\
(0.06)\end{array}$ & $\begin{array}{c}1.38 \\
(0.06)\end{array}$ & $\begin{array}{c}1.50 \\
(0.06)\end{array}$ & $\begin{array}{c}1.53 \\
(0.06)\end{array}$ & $\begin{array}{c}1.67 \\
(0.08)\end{array}$ & $\begin{array}{c}1.61 \\
(0.07)\end{array}$ & $\begin{array}{c}1.68 \\
(0.07)\end{array}$ & $\begin{array}{c}1.64 \\
(0.06)\end{array}$ \\
\hline$p$-value & 0.0020 & 0.0020 & 0.0020 & 0.0020 & 0.0020 & 0.0070 & 0.0030 & 0.0050 & 0.0070 & 0.031 \\
\hline
\end{tabular}
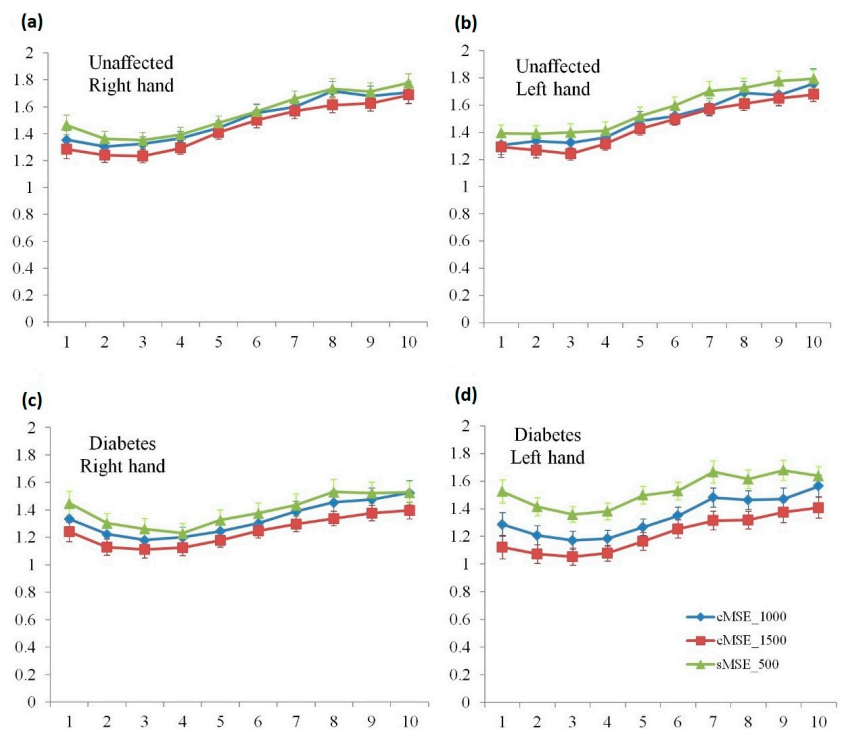

Figure 2. Comparisons between $\mathrm{SMSE}_{500}$ (solid green line), $\mathrm{cMSE}_{1000}$ (solid blue line), and $\mathrm{cMSE}_{1500}$ (solid red line) with standard errors (vertical bar) in right hand $(\mathbf{a}, \mathbf{c})$ and left hand $(\mathbf{b}, \mathbf{d})$ of the unaffected and those with diabetes. 
Figure 2 shows the comparisons between cMSE ${ }_{1500}, \mathrm{cMSE}_{1000}$, and $\mathrm{sMSE}_{500}$ at scale factors 1-10 in the right dominant hand and left non-dominant hand of the individuals with or without diabetes. There were no differences between $\mathrm{cMSE}_{1500}, \mathrm{CMSE}_{1000}$, and $\mathrm{SMSE}_{500}$ at each scale factor in the right dominant hand of the unaffected and those with type 2 diabetes, and in the left non-dominant hand of the unaffected. However, the values of $\mathrm{cMSE}_{1500}, \mathrm{CMSE}_{1000}$, and $\mathrm{SMSE}_{500}$ differed significantly from the lowest to the highest at each scale factor in the left non-dominant hand of those with diabetes.

Figure 3 shows the MSE differences between the unaffected and those with diabetes in the dominant hand and non-dominant hand. In the right dominant hand, the large-scale $\mathrm{SMSE}_{500}$, $\mathrm{CMSE}_{1000}$, and $\mathrm{CMSE}_{1500}$ values of the unaffected were significantly smaller than those of the individuals with diabetes. In contrast, in the left non-dominant hand, the large-scale $\mathrm{cMSE}_{1500}$ of the unaffected was significantly smaller than that of those with diabetes. However, the large-scale sMSE $_{500}$ and $\mathrm{cMSE}_{1000}$ of the unaffected did not differ from those of the individuals with diabetes.
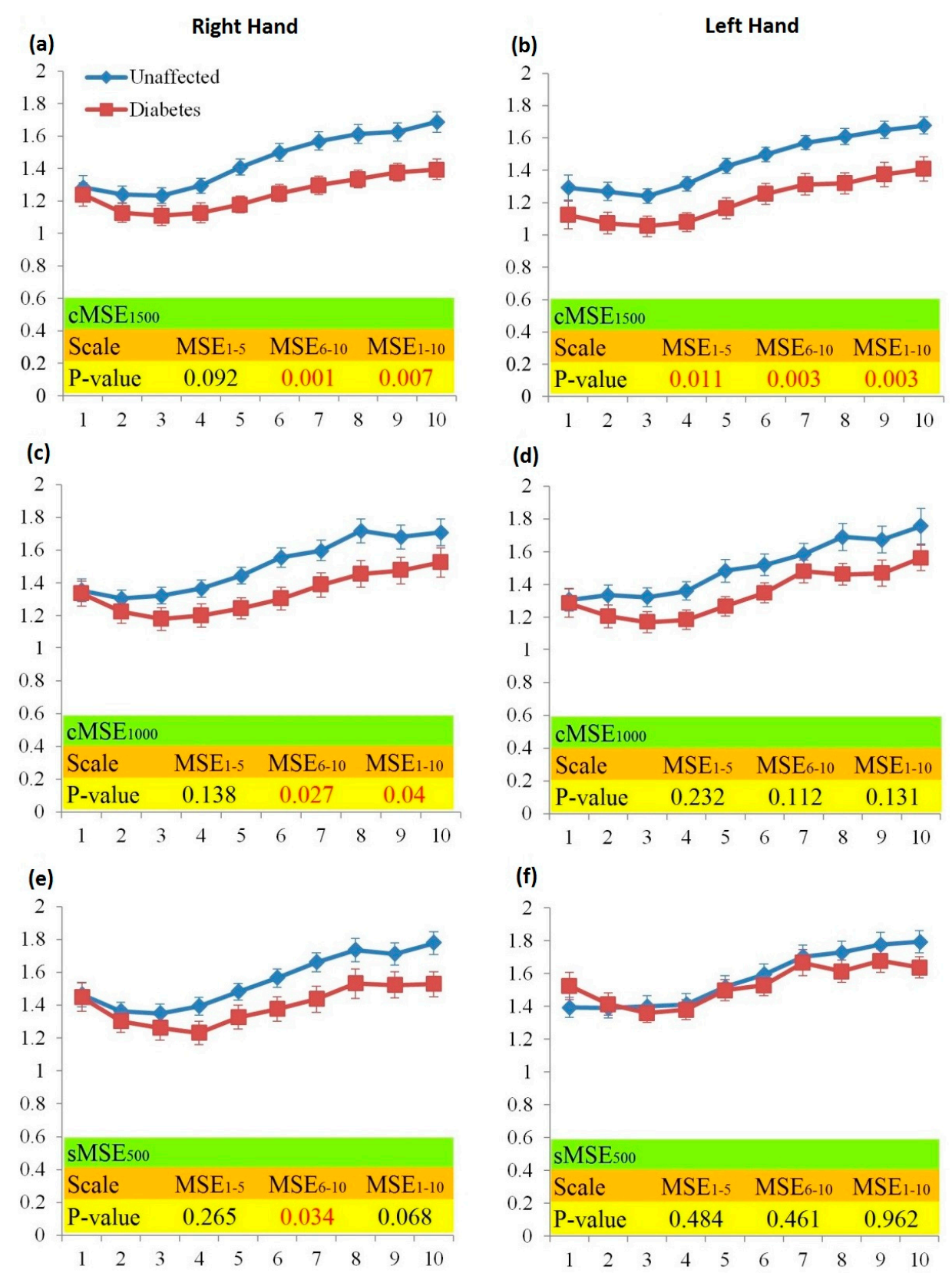

Figure 3. MSE differences between the unaffected (solid blue line) and the diabetes (solid red line) in dominant right hand $(\mathbf{a}, \mathbf{c}, \mathbf{e})$ and non-dominant left hand $(\mathbf{b}, \mathbf{d}, \mathbf{f})$. 


\section{Discussion}

Our principal findings were that in participants without diabetes, the small- and large-scale SMSE $_{500}$ and each cMSE of both the dominant and non-dominant hands did not differ significantly. In those with diabetes, the $\mathrm{SMSE}_{500}$ and each cMSE of the dominant hand were similar as well; however, both the small- and large-scale SMSE $_{500}$ and each cMSE of the non-dominant hand differed significantly. This suggested that if PPG signals were acquired from the non-dominant and physically inactive hand, the sMSE and cMSE of lower PPG signal numbers could not differentiate the unaffected individuals and those with diabetes.

Previous studies have demonstrated that poor glycemic control and physical inactivity increase the risk of microvascular disease and cardiovascular or peripheral autonomic neuropathy in diabetes [14-16]. A meta-analysis of randomized trials revealed that the risk of microvascular complications could be reduced by improving glycemic control [17]. In addition, the prevalence of microvascular complications may be reduced by exercise which improves glycemic control and endothelial function in diabetes [18-20]. The PPG pulse amplitudes of the fingertips are affected by central cardiopulmonary cycles and peripheral blood volume, pressure, and resistance [21]. Therefore, the MSE of PPG pulse amplitudes might represent the complexity of cardiovascular, respiratory, and autonomic nervous function. Both diabetes with hyperglycemia and weak handgrip strength are associated with higher risk of microvascular complications and thereby bilateral fingertip PPG pulse amplitude signals can be used for comparisons between cMSE and SMSE.

Our study clarified that the sMSE of lower PPG pulse signals from the non-dominant hand of those with diabetes was overinflated and did not differ from that of the unaffected individuals, resulting in an erroneous conclusion. To our knowledge, there is a higher probability of microvascular and autonomic nervous dysfunctions of the non-dominant physically inactive hand in diabetes. As has been shown in post-exercise heart rate recovery [22,23], those with impaired cardiac autonomic function would have a slowly declining heart beat rate at one or two minutes after cessation of the treadmill test. This may explain why more numbers of PPG pulse signals are required for the MSE analysis in a multi-comorbid condition. Although, in our study, the PPG pulse amplitudes were acquired after resting for $10 \mathrm{~min}$ in all participants, a longer time for a faster heart beat rate to slow down in those with diabetes who had a weak parasympathetic nervous activity might be necessary. The unaffected participants with a mean pulse rate of 73.4 beats per minute would take an average of $6.8 \mathrm{~min}$ to obtain 500 PPG pulse signals, whereas diabetic patients with a mean pulse rate of 81.3 beats per minute had to take only $6.1 \mathrm{~min}$. Therefore, as the same signal numbers were analyzed between the two groups, the unaffected had more resting time and might be more hemodynamically stable than that with diabetes. To avoid this time bias, more resting time prior to acquiring the PPG signals or using the same PPG measuring period rather than the same signal numbers is recommended for SMSE comparisons between groups.

The strengths of our study include the availability of large numbers of serial PPG pulse amplitude signals of bilateral fingertips and the availability of a wide range of data on other covariates. Additionally, we used case-control comparisons to lessen the effect of age, sex, and weight on the MSE index. In contrast, residual confounding may have contributed to the difference between groups.

In conclusion, diabetic autonomic function may be imprecisely evaluated by the SMSE; therefore, using more signal numbers for the cMSE analysis is recommended in such a situation.

Acknowledgments: This work was supported by the research grants from the Ministry of Science and Technology, Taiwan (Grant No. MOST 104-2221-E-259-014, and MOST 105-2221-E259-007).

Author Contributions: Gen-Min Lin and Hsien-Tsai Wu conceived and designed the experiment, and they contributed equally to the study; Hsien-Tsai Wu performed the experiment and provided the study materials; Chieh-Ming Yang and Shiao-Chiang Chu analyzed the data; Bagus Haryadi and Cheng-Chan Yang made critical viewpoints to the experiment; Gen-Min Lin wrote the paper. All author have read and approved the final manuscript.

Conflicts of Interest: The authors declared no potential conflict of interests with regard to the research, authorship, and publication of this article. 


\section{References}

1. Costa, M.; Goldberger, A.L.; Peng, C.K. Multiscale entropy analysis of complex physiologic time series. Phys. Rev. Lett. 2002, 89, 068102. [CrossRef] [PubMed]

2. Wu, S.D.; Wu, C.W.; Lin, S.G.; Lee, K.Y.; Peng, C.K. Analysis of complex time series using refined composite multiscale entropy. Phys. Lett. A 2014, 378, 1369-1374. [CrossRef]

3. Costa, M.; Peng, C.K.; Goldberger, A.L.; Hausdorff, J.M. Multiscale entropy analysis of human gait dynamics. Physica A 2003, 330, 53-60. [CrossRef]

4. Wu, S.D.; Wu, C.W.; Lee, K.Y.; Lin, S.G. Modified multiscale entropy for short-term time series analysis. Physica A 2013, 392, 5865-5873. [CrossRef]

5. Choi, Y.S. Assessment of Multiscale Information for Short Physiological Time Series. Int. J. Electr. Comput. Energ. Electron. Commun. Eng. 2016, 10, 117-120.

6. Chang, Y.C.; Wu, H.T.; Chen, H.R.; Liu, A.B.; Yeh, J.J.; Lo, M.T.; Tsao, J.H.; Tang, C.J.; Tsai, I.T.; Sun, C.K.; et al. Application of a Modified Entropy Computational Method in Assessing the Complexity of Pulse Wave Velocity Signals in Healthy and Diabetic Subjects. Entropy 2014, 16, 4032-4043. [CrossRef]

7. Yang, C.C.; Wu, H.T.; Chuang, W.Y.; Liu, T.C.; Tsai, I.T.; Chen, J.J.; Sun, C.K. Application of Short-Time MSE in Assessing Impact of Acupuncture on Peripheral Blood Flow and Autonomic Activities in Normal and Overweight Subjects. J. Med. Biol. Eng. 2016, 36, 386-395. [CrossRef]

8. Wu, H.T.; Lee, C.Y.; Liu, C.C.; Liu, A.B. Multiscale cross-approximate entropy analysis as a measurement of complexity between ECG R-R interval and PPG pulse amplitude series among the normal and diabetic subjects. Comput. Math. Methods Med. 2013, 2013, 231762. [CrossRef] [PubMed]

9. Wu, H.T.; Liu, C.C.; Lo, M.T.; Hsu, P.C.; Liu, A.B.; Chang, K.Y.; Tang, C.J. Multiscale cross-approximate entropy analysis as a measure of complexity among the aged and diabetic. Comput. Math. Methods Med. 2013, 2013, 324325. [CrossRef] [PubMed]

10. Wu, Z.; Huang, N.E.; Long, S.R.; Peng, C.K. On the trend, detrending, and variability of nonlinear and nonstationary time series. Proc. Natl. Acad. Sci. USA 2007, 104, 14889-14894. [CrossRef] [PubMed]

11. Huang, N.E.; Shen, Z.; Long, S.R.; Wu, M.C.; Shih, H.H.; Zheng, Q.; Yen, N.C.; Tung, C.C.; Liu, H.H. The empirical mode decomposition and the Hubert spectrum for nonlinear and non-stationary time series analysis. Proc. R. Soc. A 1998, 454, 903-995. [CrossRef]

12. Richman, J.S.; Moorman, J.R. Physiological time-series analysis using approximate entropy and sample entropy. Am. J. Physiol. Heart Circ. Physiol. 2000, 278, H2039-H2049.

13. Costa, M.; Goldberger, A.L.; Peng, C.K. Multiscale entropy analysis of biological signals. Phys. Rev. E 2005, 71, 021906. [CrossRef] [PubMed]

14. Klein, R.; Klein, B.E.; Moss, S.E.; Cruickshanks, K.J. Relationship of hyperglycemia to the long-term incidence and progression of diabetic retinopathy. Arch. Intern. Med. 1994, 154, 2169-2178. [CrossRef] [PubMed]

15. Bash, L.D.; Selvin, E.; Steffes, M.; Coresh, J.; Astor, B.C. Poor glycemic control in diabetes and the risk of incident chronic kidney disease even in the absence of albuminuria and retinopathy: Atherosclerosis Risk in Communities (ARIC) Study. Arch. Intern. Med. 2008, 168, 2440-2447. [CrossRef] [PubMed]

16. Young, R.J.; Ewing, D.J.; Clarke, B.F. Nerve function and metabolic control in teenage diabetics. Diabetes 1983, 32, 142-147. [CrossRef] [PubMed]

17. Hemmingsen, B.; Lund, S.S.; Gluud, C.; Vaag, A.; Almdal, T.P.; Hemmingsen, C.; Wetterslev, J. Targeting intensive glycaemic control versus targeting conventional glycaemic control for type 2 diabetes mellitus. Cochrane Database Syst. Rev. 2013, 11, 008143.

18. Maiorana, A.; O’Driscoll, G.; Cheetham, C.; Dembo, L.; Stanton, K.; Goodman, C.; Taylor, R.; Green, D. The effect of combined aerobic and resistance exercise training on vascular function in type 2 diabetes. J. Am. Coll. Cardiol. 2001, 38, 860-866. [CrossRef]

19. Fuchsjäger-Mayrl, G.; Pleiner, J.; Wiesinger, G.F.; Sieder, A.E.; Quittan, M.; Nuhr, M.J.; Francesconi, C.; Seit, H.P.; Francesconi, M.; Schmetterer, L.; et al. Exercise training improves vascular endothelial function in patients with type 1 diabetes. Diabetes Care 2002, 25, 1795-1801. [CrossRef] [PubMed]

20. Balducci, S.; Iacobellis, G.; Parisi, L.; Di Biase, N.; Calandriello, E.; Leonetti, F.; Fallucca, F. Exercise training can modify the natural history of diabetic peripheral neuropathy. J. Diabetes Complicat. 2006, 20, $216-223$. [CrossRef] [PubMed] 
21. Elgendi, M. On the analysis of fingertip photoplethysmogram signals. Curr. Cardiol. Rev. 2012, 8, 14-25. [CrossRef] [PubMed]

22. Okutucu, S.; Karakulak, U.N.; Aytemir, K.; Oto, A. Heart rate recovery: A practical clinical indicator of abnormal cardiac autonomic function. Expert Rev. Cardiovasc. Ther. 2011, 9, 1417-1430. [CrossRef] [PubMed]

23. Banthia, S.; Bergner, D.W.; Chicos, A.B.; Ng, J.; Pelchovitz, D.J.; Subacius, H.; Kadish, A.H.; Goldberger, J.J. Detection of cardiovascular autonomic neuropathy using exercise testing in patients with type 2 diabetes mellitus. J. Diabetes Complicat. 2013, 27, 64-69. [CrossRef] [PubMed]

(C) 2017 by the authors. Licensee MDPI, Basel, Switzerland. This article is an open access article distributed under the terms and conditions of the Creative Commons Attribution (CC BY) license (http:/ / creativecommons.org/licenses/by/4.0/). 